\title{
Prosocialidad en niños, niñas y adolescentes desvinculados de grupos armados ilegales: retos y reflexiones para la investigación social ${ }^{*}$
}

\author{
Prosociality in separated children and teenagers \\ of illegal armed groups: Challenges and reflections \\ in social research.
}

Anyerson Stiths Gómez Tabares** Universidad Católica Luis Amigó, Colombia

Mariela Narváez Marín Universidad de Manizales, Colombia

Recibido: 1 de agosto de 2017 Revisado: 14 de octubre de 2017 Aceptado: 24 de enero de 2018

\section{Resumen}

Este articulo constituye una primera reflexión teórica de la investigación titulada "Conductas prosociales en niños, niñas y adolescentes desvinculados de grupos armados ilegales, pertenecientes a la modalidad Hogar Sustituto Tutor del Instituto Colombiano de Bienestar Familiar, en la ciudad de Manizales". Se busca, en primer lugar, hacer un rastreo de la categoría prosocialidad, sus principales referentes históricos, conceptuales e investigativos. Posteriormente, se plantean hipótesis y propuestas alternativas, ubicando la categoría de potencial prosocial como eje central para la investigación y el trabajo con niños, niñas y adolescentes desvinculados de grupos armados ilegales que están en procesos de reincorporación a la vida civil.

El presente artículo hace parte del proyecto de investigación: Conductas prosociales en niños, niñas y adolescentes desvinculados de grupos armados ilegales, pertenecientes a la modalidad Hogar Sustituto Tutor del Instituto Colombiano de Bienestar Familiar, en la ciudad de Manizales, el cual tuvo una duración de 12 meses (enero- diciembre del 2017). Financiado por la Universidad Católica Luis Amigó, Manizales, Colombia, con alianza de cooperación de la Universidad de Manizales, Colombia. El proyecto está vinculado al grupo de investigación Estudios de Fenómenos Psicosociales, Universidad Católica Luis Amigó, Medellín, Colombia. DOI: https://doi.org/10.15332/s1794-9998.2018.0002.05 Correspondencia: Anyerson Stiths Gómez Tabares, Docente e Investigador, Universidad Católica Luis Amigó. Correo electrónico: anyerspn. gomezta@amigo.edu.co; anyerson_1988@hotmail.com . Dirección postal: Calle 57 No 21- 25, Manizales, Colombia 
Palabras clave: Prosocialidad, grupos armados ilegales, niñez y adolescencia, empatía, juicio moral, reintegración.

\begin{abstract}
This article is a first theoretical reflection of a study entitled "Prosocial behaviors in children and adolescents separated from illegal armed groups" carried out at Hogar Tutor of the Colombian Institute for Family Welfare, in Manizales. It aims to trace prosociality, its main historical, conceptual and research antecedents, and then hypotheses and alternative proposals are set forth, placing the category of prosocial potential as the central axis for research and work with children and adolescents who are not related to illegal armed groups, and who are in the process of reincorporation into civilian life.
\end{abstract}

Keywords: prosociality, illegal armed groups, children and adolescents, empathy, moral judgment, reintegration.

\section{Introducción}

El estudio de la conducta prosocial surge en el campo de la psicología social (Caprara, Steca, Zelli, \& Capanna, 2005), el cual se ha logrado posicionar como un área de estudio prometedora para las ciencias sociales tanto para la investigación como la intervención, especialmente en escenarios de infancia y juventudes. Su estudio ha tenido un inicio reciente en la psicología, pues tradicionalmente la conducta agresiva y antisocial ha tenido un papel protagónico en la investigación (Eisenberg \& Morris, 2004), motivo por el cual, se considera importante hacer una revisión del soporte conceptual de la categoría de prosocialidad, su desarrollo histórico, las variables relacionadas y las principales tendencias investigativas y teóricas.

Además de esta revisión, se busca construir una reflexión conceptual respecto a los vacíos, retos y alcances de la investigación y la intervención social en materia de prosocialidad en niños, niñas y adolescentes desvinculados de grupos armados ilegales. Es de mencionar que, son mínimos los referentes investigativos de este constructo en esta población a pesar del contexto social actual en el país en términos de construcción de paz y reconciliación.

Finalmente, se introduce la noción de potenciales prosociales y se plantean algunas ideas para la formulación de una propuesta alternativa, tanto para la investigación como para la intervención, con niños, niñas y adolescentes desvinculados de grupos armados que están en procesos de reincorporación a la vida social.

\section{La conducta prosocial}

\section{¿Qué es la conducta prosocial?}

Existe una gran diversidad de definiciones y distinciones respecto al comportamiento prosocial. Vaughan \& Hogg (2010) establecen que “el comportamiento prosocial es una categoría amplia que se refiere a los actos valorados positivamente por la sociedad" (p. 528). Según Eisenberg, Cumberland, Guthrie, Murphy \& Shepard (2005), la conducta prosocial debe tener la intención de beneficiar o ayudar a otras personas. Para Mestre (Mestre, Samper \& Frías, 2002; Mestre, Tur, Samper, Nácher \& Cortés, 2007) y Martí Vilar (2010), una de sus características es que debe ser voluntaria, además de ser un comportamiento valorado positivamente por la sociedad y que conduce, por tanto, a consecuencias sociales positivas.

La Conducta Prosocial está determinada por actos intencionales (Martí Vilar, 2011) dirigidos a llevar a cabo comportamientos tales como ayudar a los 
demás, compartir, cooperar, trabajar en equipo, intercambiar lenguajes afectivos, tomar en cuenta al otro y retroalimentarlo positivamente frente a lo que dice o hace (Ministerio de Protección Social, 2007).

Lo anterior, muestra una multiplicidad de significados y variables asociadas que se pueden encontrar en la literatura científica, esto se debe, en gran parte, al nivel de detalle y el número de variables que implica el término (Auné, Blum, Abal, Lozzia \& Horacio, 2014). Una muestra de esto es la dificultad para unificar criterios respecto a si se deben incluir o no variables motivacionales en su definición.

Por su parte, Martí Vilar (2011) establece dos tipos de definiciones: las conductuales y las motivacionales. La primera, considera homónimos los conceptos de prosocialidad y altruismo; reconoce, en el sentido amplio, que toda conducta prosocial siempre está orientada al beneficio de los demás. La segunda, hace una distinción entre la conducta prosocial y el altruismo; reconoce que una conducta prosocial puede orientarse a ayudar a los demás, pero también pueden existir conductas prosociales al servicio del beneficio propio.

Se considera importante hacer la distinción entre el comportamiento prosocial, comportamiento de ayuda y altruismo, pues en ocasiones son tomados como términos intercambiables en la literatura científica. El comportamiento de ayuda es considerado una subcategoría del comportamiento prosocial (Gómez \& Gaviria, 2007; Martí Vilar, 2010; Vaughan \& Hogg, 2010), y se refiere a actos intencionales que buscan beneficiar a otra persona o grupo de ellas.

El altruismo se refiere a un acto motivado por el deseo de beneficiar a un tercero más que así mismo (Batson, 1991; Batson, \& Powell, 2003; Vaughan \& Hogg, 2010). Auné et al. (2014) plantean que "La conducta altruista es toda acción voluntaria realizada con la intención de ayudar a los demás, provocando o manteniendo efectos positivos" (p. 22). Por el contrario, para Batson, \& Powell (2003) la conducta prosocial puede estar motivada también por factores diferentes al altruismo, por ejemplo, motivaciones colectivistas, egoístas, morales-normativas, entre otras, y aun así considerarse prosocial dada las consecuencias positivas que genera. En este sentido, la prosocialidad puede implicar o no una motivación altruista (González-Portal, 2000).

También se ha considerado la empatía como una subcategoría estrechamente relacionada con la conducta prosocial (Fernández Pinto, López-Pérez, \& Márquez, 2008), debido a que "la empatía involucra no sólo la experiencia afectiva del estado emocional real o inferido de otra persona, sino, además, algún reconocimiento y comprensión mínimos del estado emocional del otro" (Richaud de Minzi, 2009, p. 188). Esta experiencia afectiva o reacción emocional debe ser congruente con el estado emocional de otra persona o al menos muy similar con la experiencia subjetiva y sentir del otro. (Eisenberg, Zhou \& Koller, 2001; Eisenberg, Valiente \& Champion, 2004).

En este sentido, hay una relación proposicional entre la empatía y la conducta prosocial, siendo la primera un predictor para la segunda, a la vez que es un generador del comportamiento altruista (Graziano, Habashi, Sheese \& Tobin, 2007; Gutiérrez San Martín, Escartí \& Pascual, 2011).

En general, es posible afirmar que la conducta prosocial es un concepto multidimensional, compuesto por una diversidad de variables positivamente asociadas tales como: conductas de ayuda, altruismo y empatía por el beneficio de otras personas; involucra, además, diversos factores motivacionales. En últimas, se puede considerar que toda conducta prosocial es una conducta social positiva.

\section{Referente histórico en el estudio de la conducta prosocial}

\section{Primeros referentes teóricos}

La investigación sobre la conducta prosocial surge en el campo de la psicología social (Caprara et al, 2005; Roche, 2010). Este concepto es, además, una antítesis de la conducta antisocial. Existe una gran variedad de referentes teóricos y fenómenos 
sociales que dieron origen a esta categoría como objeto de estudio.

Uno de los referentes de mayor aceptación en la literatura científica se dio en los años cincuenta, tras el asesinato de Kitty Genovese en New York (Carrera, Caballero \& Oceja, 2003; Moñivas, 1996; Vaughan \& Hogg, 2010), suceso que atrajo la atención de investigadores y psicólogos sociales sobre el estudio del comportamiento de ayuda en situaciones de emergencia.

El enfoque de estas investigaciones fueron los factores situacionales que influenciaban la intervención del espectador ante una emergencia, es decir, el potencial socorrista de una persona (Baron \& Byrne, 2005; Dovidio, Piliavin, Schroeder \& Penner, 2006; Gómez \& Gaviria, 2007). Como lo establece Vaughan \& Hogg (2010) “Lo que hoy se sabe es que es más probable que el espectador solitario ayude a que lo haga uno de entre varios espectadores, fenómeno conocido por el efecto espectador" (p. 541).

Según González (1992, citado por Martí Vilar, 2011) el interés científico por las conductas prosociales se dio en la década de los setenta, tratando de encontrar otras alternativas a las conductas agresivas, delictivas y antisociales; así como tratar de hallar explicaciones a la inhibición social que se presentaba ante diferentes situaciones de emergencia por los demás. Las investigaciones sobre el efecto espectador permitieron el desarrollo de dos líneas teóricas para explicar el comportamiento prosocial: el modelo cognitivo de Latané y Darley (Latané, 1981) y el modelo de los costos de la ayuda de Piliavin, \& Charng (1990). Ambos buscaban explicar el comportamiento de ayuda y las razones por las cuales una persona no socorría $a$ alguien en situaciones de emergencia.

El primer modelo plantea que la falta de ayuda, como fue el caso de Kitty Genovese, se daba cuando el tamaño del grupo de espectadores aumentaba, lo cual indicaba que, a mayor número de espectadores menor es la probabilidad de ayuda (Latané, 1981; Latané \& Darley, 1970; Latané \& Darley, 1976). Hay dos elementos que explican el fenómeno: la difusión de responsabilidad y el temor a las torpezas sociales.
La difusión de responsabilidad indica una tendencia del espectador a pensar que los demás asumirán la responsabilidad y brindarán ayuda. Si este proceso cognitivo es asumido por todos los espectadores, es probable que ninguno brinde ayuda a la víctima (Latané \& Darley, 1976). Así, entre más espectadores, mayor es la difusión de responsabilidad. Por otra parte, el temor a las torpezas sociales explica la tendencia a la inhibición por la presencia de los espectadores (Latané, 1981; Latané \& Darley, 1976). El proceso cognitivo se da en función a una serie de ideas y temores relacionados con hacer el ridículo, actuar de manera inapropiada o cometer un error (Gómez \& Gaviria, 2007). Estos elementos inhiben la respuesta de los espectadores a actuar ante una emergencia o a hacerlo de manera efectiva.

El segundo modelo, explica el proceso cognitivo y fisiológico del espectador para evaluar el costo o beneficio de ayuda a otras personas. Algunos investigadores sociales (Dovidio et al, 2006; Piliavin \& Charng, 1990) consideran que cuando una persona presencia una situación en la cual alguien requiere de ayuda, o está en problemas, realiza una serie de cálculos antes de responder al estímulo. El proceso se da de la siguiente manera: Primero, se da una activación fisiológica y conductual, la cual está estrechamente asociada a la empatía. La activación fisiológica que se genera al percibir la angustia de una persona en problemas es, en últimas, una respuesta empática. Segundo, la activación conductual es procesada y despierta una emoción, sea miedo, angustia o enojo; el acto de ayudar a otro es una manera de reducir la ansiedad propia y también está relacionado con la empatía. Tercero, se evalúan las consecuencias de ayudar a la persona en problemas y de acuerdo con la relación costo-beneficio se elige la acción de ayudar o no. Esto quiere decir que, a mayor percepción de costos negativos menor será la posibilidad de brindar ayuda.

\section{Otras tendencias teóricas e investigación en prosocialidad}

Además de las aproximaciones teóricas e investigativas en psicología social sobre el comportamiento 
de ayuda tras la tragedia de Kitty Genovese, y los diferentes momentos históricos por los que ha pasado su estudio, surgen nuevas reflexiones y posturas respecto a los factores causales de la conducta prosocial. El debate entre el enfoque biológico versus el ambiental se ubica como objeto de investigación; la controversia sobre las disposiciones biológicas a la prosocialidad y los procesos de crianza y aprendizaje social, continúan siendo objeto de revisión teórica (Auné et al., 2014; Gómez, 2017; Correa, 2017).

El argumento de que la conducta prosocial y el altruismo tienen valor evolucionista para la supervivencia de la especie, siendo la cooperación, la reciprocidad y el mutualismo condiciones biológicas para la prosocialidad, como consideran algunos psicólogos sociales evolucionistas y sociobiólogos (Buss \& Kenrick, 1998; Buss \& Reeve, 2003; Dugatkin, 2007; Stevens, Cushman \& Hauser, 2005) resulta hoy en día insuficiente para explicar el comportamiento de ayuda y la prosocialidad. "Un problema con la teoría evolucionista como única explicación para el altruismo es la falta de pruebas humanas convincentes [...] un caso como la falta de ayuda a Kitty Genovese es difícil de explicar a nivel biológico" (Vaughan \& Hogg, 2010, p. 532).

Las explicaciones de naturaleza ambiental relacionadas con el aprendizaje social, la crianza, la socialización, la exposición a modelos de comportamiento y los enfoques motivacionales han ganado un gran número de adeptos en psicología social para explicar la conducta prosocial, especialmente, en los contextos de primera infancia, parentalidad y escenarios educacionales (Bandura, 1987; Bandura \& Walters, 1990; Garaigordobil, 2005; Richaud de Minzi, 2009).

A raíz de estos debates y reflexiones teóricas e investigativas se generan tres enfoques considerados actualmente los más representativos para la investigación de la conducta prosocial: el enfoque evolucionista, el enfoque cognitivo- evolutivo de corte moral y el enfoque del aprendizaje social.

\section{Retos para la investigación social en prosocialidad en el marco del contexto social actual}

La investigación actual reconoce tres dimensiones de la conducta prosocial, a saber, los comportamientos empáticos, el altruismo y compartir (Auné et al., 2014; Martí Vilar \& Lorente, 2010). Sin embargo, son diversas las líneas de investigación en prosocialidad que establecen una diversidad mucho más amplia de variables positivamente asociadas. Autores como Caprara (Caprara et al., 2005; Caprara et al., 2012), Marti Vilar (2011), Pastorelli (2015), Richaud de Minzi (2011), Eisenberg (Eisenberg, Fabes, Guthries, \& Reiser, 2000; Eisenberg et al, 2001) y Mestre (Mestre et al, 2002; Mestre et al., 2007) han establecido que variables como la capacidad de autocontrol, el razonamiento moral, contextos sociales positivos, creencias de autoeficacia, ajuste psicológico, empatía, altruismo, compasión, edad, género, modelos de referencia, crianza y la parentalidad son determinantes en la investigación social, y deben considerarse predictores de la conducta social positiva, además de reconocer factores motivacionales y de personalidad asociados.

Estos autores reconocen la influencia recíproca entre factores relacionados con el aprendizaje, el contexto social-familiar y elementos individuales en el estudio de la conducta prosocial; especialmente en la primera infancia, la niñez y la adolescencia en diversidad de contextos: educativos, comunitarios y de parentalidad. Sin embargo, a pesar de que estos campos investigativos han sido exitosos y continúan siendo prometedores para el trabajo en prosocialidad, son escasos los desarrollos teóricos e investigativos en etapas diferentes a la niñez y la adolescencia y en contextos sociales complejos. Como concluyen Auné et al., (2014) en su revisión del estado actual en investigación sobre la conducta prosocial:

[...] Resulta contrastante la gran cantidad de investigaciones acerca de la conducta prosocial en la infancia y en la adolescencia, con los escasos desarrollos enmarcados en etapas posteriores [...] el estudio de dicho constructo en la adultez y la vejez es un campo 
incipiente, con una gran variedad de aristas por explorar (p. 29).

De estos hallazgos resulta la necesidad de ampliar los marcos investigativos en prosocialidad, utilizar los desarrollos actuales y plantear nuevos interrogantes que, de alguna manera, permitan construir una perspectiva en el marco del contexto social actual. La reflexión sobre las posibilidades de estudiar la conducta prosocial y variables como la empatía, el altruismo o la capacidad de ayuda, en personas inmersas en contextos sociales de violencia y alta vulnerabilidad, aún está por hacer.

En este sentido, nace la reflexión sobre la posibilidad de estudiar estas variables en niños, niñas y adolescentes que han sido desvinculados, por diversos motivos, de grupos armados ilegales, y que están en procesos de reintegración a la vida civil y la sociedad.

Los contextos de referencia de esta población generalmente han estado marcados por experiencias de violencia, pautas de interacción social, familiar y psicológicas disfuncionales, además de modelos de comportamiento antisocial (Blom \& Pereda, 2009; Moreno, 2009; Pécaut, 2001; Rethmann, 2010; Springer, 2012), por lo cual resulta un reto hablar de prosocialidad en estos casos. Sin embargo, aún no hay suficiente evidencia empírica que indique que no sea posible, en primer lugar, identificar actitudes y conductas prosociales, y segundo, estimular, mediante las estrategias, programas o intervenciones adecuadas, los potenciales morales prosociales hasta el punto de desarrollar comportamientos valoradas positivamente por la sociedad.

A pesar de la gran relevancia de los estudios en categorías sobre agresividad, conducta antisocial, impulsividad y violencia en población de adolescentes y jóvenes en contextos de riesgo y vulneración, no aporta a las posibilidades de transformación desde los potenciales del sujeto $y$, por tanto, se hace imperante realizar lecturas diferentes en el campo de la investigación social que apuesten a las posibilidades de transformación del sujeto, especialmente en un terreno tan inexplorado como lo es la prosocialidad en niños, niñas y adolescentes desvinculados de grupos armados. En otras palabras, una apuesta al estudio de los potenciales prosociales de esta población y no a una mirada del déficit.

\section{Otras posibilidades de estudio}

Desde un punto de vista teórico, las contribuciones desde los enfoques cognitivo- evolutivos de corte moral de Turiel (1979), Kohlberg (Kohlberg, 1976, 1987, Kohlberg , Power \& Higgins, 2002) y Rest (Rest, 1986; Rest, Narvaez, Bebeau \& Thoma, 1999) señalan la posibilidad de estimular el razonamiento moral prosocial mediante estrategias educacionales y prácticas sociales favorables. Para el caso de la niñez y la adolescencia, el desarrollo moral está asociado a prácticas de socialización positivas que facilitan la toma de perspectiva y la construcción autónoma de reglas.

Al respecto, las posturas del aprendizaje social de Bandura (1987) resaltan la importancia del modelado de conductas empáticas y prosociales por parte de las figuras de cuidado parental en el aprendizaje de conductas prosociales en el niño. Diversos estudios (Carlo, McGinley, Hayes, Batenhorst \& Wilkinson, 2007; Carlo, Mestre, Samper, Tur \& Armenta, 2010; Mestre et al., 2003; Eisenberg \& Fabes, 1998; Eisenberg et al, 2004; Richaud de Minzi et al., 2011) señalan que la conducta prosocial es el resultado de los estilos de crianza parental, siendo el modelado el principal mecanismo precursor para el desarrollo de comportamientos prosociales en los niños. "La conducta prosocial emerge en el segundo año de vida de los niños y se incrementa en frecuencia y variedad durante los años siguientes" (Kärtner \& Keller, 2010, p. 905).

El punto problematizador que se desea posicionar, y que además ha sido poco explorado, reside en considerar la posibilidad de procesos de re-modelamiento y re-estimulación moral en jóvenes que han tenido una exposición temprana a modelos antisociales y desarrollos morales instrumentales, mediante su ubicación en contextos protectores con figuras positivas de cuidado y, exponiéndolos a estrategias educacionales basadas 
en la prosocialidad. La hipótesis sería que, cuando un niño o adolescente es retirado de un contexto familiar y/o social violento tiene mayores posibilidades de adquirir comportamientos socialmente positivos si se ubica en un escenario social, familiar y educacional prosocial, y además, es expuesto a modelos positivos de comportamiento a no hacerlo. En este caso, de ser posible, sería necesario estudiar el nivel de prosocialidad adquirido mediante estos procesos de re-modelamiento y re-estimulación moral prosocial.

Lo cierto es que, la investigación empírica sobre la influencia de figuras de cuidado, la estimulación moral y el modelado en el desarrollo de la empatía y la conducta prosocial en etapas tardías del desarrollo, posterior a experiencias socializadoras agresivas, es escasa y aún no ofrece un soporte empírico definitivo.

\section{Una apuesta alternativa para la investigación e intervención a la niñez y la adolescencia en procesos de reintegración social}

En este apartado se busca reflexionar en torno a la atención a niños, niñas y adolescentes desvinculados de grupos armados ilegales que están en diversas medidas de protección del Estado, especialmente aquellos pertenecientes a la modalidad Hogar Sustituto Tutor del ICBF, resaltando la prosocialidad como eje central para la investigación social y la generación de propuestas de intervención que aporten a la construcción del lazo social, la superación del trauma, la reincorporación a la vida civil y la construcción de paz en contextos de pos-acuerdo en esta población.

La conducta prosocial, como ya se indicó, se desarrolla en las primeras relaciones de apego parental, con influencia del contexto social y educativo, y ciertos determinantes psicológicos que posibilitan el desarrollo de la prosocialidad (Carlo et al, 2010; Martí Vilar, 2011; Richaud de Minzi, 2009). Sin embargo, en el caso de los niños, niñas y adolescentes desvinculados de grupos armados se ha configurado en contextos de alta vulnerabilidad psicosocial, exposición a eventos violentos y potencialmente traumáticos, los cuales están asociados al desajuste psicológico y diversas afectaciones emocionales (Blom \& Pereda, 2009; Gutiérrez, 2015; Springer, 2012). Como lo afirman Mestre, Samper, Tur, Cortés y Nácher (2006), “estudios realizados en la infancia y la adolescencia han concluido que bajos niveles de conducta prosocial constituyen un factor de riesgo de problemas de conducta y desórdenes afectivos" (p. 203). De igual manera, como lo plantea Bandura (citado por Richaud de Minzi, 2014):

los mecanismos específicos de socialización han sido relacionados con la adquisición de nuevos comportamientos. Los niños que han sido expuestos al modelado de comportamientos específicos tendrán más probabilidad de repetir esos actos (especialmente si el modelo es admirado o se está íntimamente identificado con él (p. 174).

Todas estas formas de vulneración a nivel psicológica, social, cultural, e incluso familiar, son barreras multidimensionales para el desarrollo de conductas prosociales y configuran formas de interacción basadas en el miedo y la violencia, además de la alienación y sometimiento que genera el grupo armado sobre los niños y adolescentes, anulando la posibilidad de juicios ético-morales diferentes a los determinados por las ideológicas impuestas. (Kimmel \& Roby, 2007; Maclurea \& Denov, 2006; Moreno, 2009; Springer, 2012)

A pesar de todas estas barreras, una lectura desde el potencial humano indicaría que el niño, niña o adolescente, al ser desvinculado de grupos armados ilegales, puede aprender, re-aprender y remodelar conductas sociales positivas coherentes con sus contextos protectores (Mago, 2009; Moreno, 2009; Romero \& Chávez, 2008). Esto indica que, al ser ubicados en medidas de protección, específicamente modalidades familiares sustitutas tutoras del Instituto Colombiano de Bienestar Familiar, se estaría reconociendo, implícitamente, la prosocialidad como epicentro del proceso de reincorporación a la vida civil y la construcción del lazo social.

El punto central de esta reflexión es el reconocimiento de un potencial prosocial posible de ser 
amplificado mediante estrategias de intervención estructuradas y escenarios sociales protectores, encaminados al lazo social y al desarrollo de conductas sociales positivas. Dicho potencial y su posibilidad de estimulación, se ubica como eje de la investigación social en prosocialidad con esta población que abre, además, un panorama valioso para la intervención (Gómez, 2017).

Un elemento que apoya la tesis de los potenciales prosociales son las inquietudes morales que expresan los adolescentes ubicados en hogares sustitutos tutores sobre la injusticia social, la protección por sus iguales y el cuestionamiento de ideales sociales (Blom \& Pereda, 2009; Cortes \& Buchanan, 2007; Echeburúa, 2004; Gómez, 2017; Rethmann, 2010).

Estas inquietudes y malestares morales pueden encontrar vías impulsivas de expresión no coherentes con la prosocialidad (Rethmann, 2010; Blom \& Pereda, 2009) y, por tanto, no pueden considerarse estrictamente como una conducta prosocial, sin embargo, es un referente moral importante, el cual se puede considerar un potencial prosocial posible de ser amplificado y estimulado bajo procesos de investigación e intervención interdisciplinar.

La falta de una mayor evidencia empírica al respecto exige la necesidad de investigar dichos potenciales prosociales en esta población, lo cual contribuiría, al mismo tiempo, al diseño de estrategias, programas y metodologías de trabajo más eficaces que permitan el fortalecimiento de la prosocialidad en esta población.

De acuerdo con lo problematizado hasta ahora, surgen dos propuestas alternativas que pretenden conjugar la investigación y la intervención como dos niveles de reflexión, construcción teórica y dar soporte empírico al estudio de la prosocialidad en niños, niñas y adolescentes desvinculados de grupos armados ilegales, máxime cuando son mínimos los referentes investigativos, teóricos y prácticos de la relación entre prosocialidad y víctimas de la confrontación armada.

\section{Las propuestas son las siguientes:}

La recuperación de la confianza básica como elemento constitucional del re-modelamiento prosocial

Esta tesis parte de la existencia de un potencial prosocial en el niño, niña o adolescente desvinculado, el cual solo podría desarrollarse mediante procesos de recuperación de la confianza básica en el otro. La confianza es la categoría fundamental para el re- modelamiento prosocial en esta población (Correa, 2017; Gómez, 2017; Gómez \& Narváez, 2017). La recuperación de la confianza básica y la empatía son, en sus inicios, los precursores del desarrollo de la conducta prosocial y, por tanto, una base valiosa para el modelamiento prosocial (Caprara et al., 2005; Caprara et al., 2012; Eisenberg et al., 2000; Eisenberg \& Morris, 2004; Mestre et al., 2007; Mestre et al., 2004). Este elemento es indispensable para la adaptación y la reincorporación de esta población a la vida familiar y social.

La confianza y la empática son variables positivamente asociadas a la conducta prosocial (Eisenberg et al., 2001; Mestre, et al., 2009; Pastorelli, 2015), debido a que "la empatía tiene que ver con la condición de las personas de ubicarse en el lugar del otro y comprender sus acti-tudes y sus estados mentales" (Parra, 2002, p. 115), además del reconocimiento del estado emocional de la otra persona.

Diversos estudios (Baita, 2015; Blom \& Pereda, 2009; Echeburúa, 2004; Greenwald, 2005; ICBF, OIM y Unicef, 2014; Machel, 1996) ubican la pérdida de la confianza básica como una reacción psicológica a la exposición traumática de la guerra o algún tipo de violencia interpersonal. Si la conducta prosocial está determinada, en parte, por procesos de socialización, modelamiento de referentes prosociales y estimulación educacional, estos no lograrán ninguna influencia en el niño o adolescente en la medida en que no es posible la elaboración emocional, el aprendizaje de modelos prosociales ni el aprendizaje de nuevas formas de relacionamiento social sin la base de la confianza en los demás. 
De la misma manera que la empatía es una subcategoría de la conducta prosocial, la confianza básica en el otro es un precursor y una condición necesaria para la empatía. Los estudios sobre el apego de Bowlby $(1976,1986)$ rescatan la importancia de construir una base segura en la configuración de la confianza en el otro. De aquí se parte para plantear que las posibilidades de exponer a esta población a modelos prosociales de cuidado y re-modelar conductas y actitudes sociales positivas requiere de la confianza.

Esta propuesta implica dos apuestas investigativas y de intervención. Por un lado, estudiar el nivel de afectación psicológica en términos de la pérdida de la confianza básica en el otro, y establecer su relación con la empatía como precursor de la conducta prosocial. Por otro lado, implicaría nuevas apuestas de intervención para el desarrollo de conductas prosociales. Así como lo plantea Gómez (2017):

Cuando los niños, niñas o adolescente logran desarrollar sentimientos de confianza, sensibilidad social por el bienestar de sus cuidadores, educadores, madres sustitutas tutoras, equipo psicosocial y otras figuras que están en los procesos de protección, reducen las manifestaciones de agresividad, adquieren la capacidad de comprender las consecuencias potencialmente negativas para ellos mismo y los otros. (p. 1502)

\section{La estimulación del juicio moral como estrategia de fortalecimiento de la prosocialidad}

Esta propuesta nace como resultado de los hallazgos investigativos con población adolescente (Carlo et al, 2010; Eisenberg \& Morris, 2004; Richaud de Minzi; 2009), donde establecen la relación entre el desarrollo moral y la conducta prosocial. De acuerdo con Martínez, Barreto, Duran y Castro (2014), a mayor nivel de razonamiento moral mayor es la tendencia prosocial, por el contrario, un menor nivel razonamiento moral prosocial estará ligado a relaciones instrumentales, la agresividad y conductas externalizantes. Al respecto, los autores concluyen que:
A medida que se incrementa el estadio del desarrollo moral, se disminuyen las conductas de agresión tales como burla, agresión física y bullying social (p. 70) [...] Por el contrario, mientras menor sea el nivel de desarrollo moral de una persona, dado que su razonamiento moral se halla en los estadios más bajos de los planteados por Kohlberg, las conductas agresivas se incrementan, lo mismo que la posibilidad de ser agresor (p. 71)

Esta relación se ha encontrado en diversas investigaciones realizadas con niños y adolescentes (Carlo et al, 2010; Hemming, 1991; Hoffman, 1992; Holmgren, Eisenberg \& Fabes, 1998; Richaud de Minzi, 2009), motivo por el cual, se considera la importancia de la estimulación moral en esta población, a la vez que será un posibilitador de la empatía y el lazo social.

La estimulación moral es un proceso complejo asociado a factores cognitivo-evolutivos, que se han configurado por influencia de la experiencia e historia del sujeto, prácticas de socialización y relaciones cercanas de los niños, niñas y adolescentes con sus figuras parentales y de protección (Henao, Ramírez \& Ramírez, 2007; Kohlberg, 1969; Martí Vilar, 2011; Ramírez, 2007; Richaud de Minzi, 2009). En este sentido, son diversas las barreras que se han configurado en términos de desarrollo moral prosocial en la población desvinculada de grupos armados, justamente por la exposición temprana que han tenido a eventos de guerra, violencia y sometimiento moral. Sin embargo, la evidencia empírica ha demostrado que es posible, especialmente en la niñez y la adolescencia, estimular el juicio moral mediante la implementación de estrategias de acompañamiento psicoeducativo en contextos de protección y generadores de confianza y afecto.

De acuerdo con el modelo cognitivo evolutivo (Kohlberg et al, 2002; Kohlberg, Levine \& Heder, 1992), la estimulación ético-moral responde a estrategias de intervención dialógica y educacional, pues se considera que su desarrollo no es un proceso estático ni evolutivamente determinado. De esta manera, el desarrollo de los potenciales prosociales en niños, niñas y adolescentes 
desvinculados de grupos armados, ubicados en medidas de protección, solo es posible mediante el reconocimiento de sus potenciales, la recuperación de la confianza, la implementación de estrategias psicosociales y psicoeducativas adecuadas, que posibiliten las condiciones para la reflexión ética, la elaboración emocional y el acceso a formas más complejas de razonamiento moral orientadas a la prosocialidad.

Lo anterior, abre un campo de investigación - acción en prosocialidad con esta población en la medida en que se hace necesario soportar empíricamente la posibilidad de fortalecer actitudes y conductas prosociales mediante estrategias basadas en la psicoeducación y estimulación del juicio moral.

Finalmente, La prosocialidad debe considerarse una categoría valiosa de investigación e intervención a la niñez y adolescencia desvinculada de grupos armados ilegales, que están en procesos de reintegración a la vida civil, pues solo desde la perspectiva del potencial humano sería posible la trasformación social en el país y la resignificación en tiempos de pos-acuerdo. Trabajar los potenciales prosociales exige, como lo plantea Gómez (2017):

el ejercicio de trasformar la figura a veces de combatiente, huérfano o víctima, en que se halla la constitución discursiva e identitaria del niño, niña o adolescente, a la figura del ciudadano, aquel que trasciende la relación instrumental al vínculo prosocial, la personalidad adaptativa en busca de la supervivencia a una estructuración del yo que integre y reconozca su emocionalidad y su accionar en el mundo, recupere la confianza en el otro como fundamento de la prosocialidad y reconozca la otredad como elemento ético y ciudadano (p. 1505).

\section{Referencias}

Auné, S. E., Blum, D., Abal, J. P., Lozzia, G. S. \& Horacio, F. A. (2014). La Conducta Prosocial: Estado actual de la investigación. Pers- pectivas en Psicología: Revista de psicología y ciencias afines, 11(2), 21-33.

Baita, S. (2015). Rompecabezas. Una guía introductoria al trauma y la disociación en la infancia. Buenos Aires: editorial Ciudad Autónoma de Buenos Aires

Bandura, A. (1987). Teorías del Aprendizaje Social. Madrid: Espasa

Bandura, A., \& Walters, R. (1990). Aprendizaje social y desarrollo de la personalidad. Madrid: Espasa

Baron, R. A., \& Byrne, D. (2005). Psicología Social. $10^{\mathrm{a}}$ ed. Madrid: Pearson

Batson, C. D. (1991). The altruism question: Toward a Social Psychological Answer. Hillsdale, NJ: Erlbaum.

Batson, C. D. \& Powell, A. (2003). Altruism and prosocial behavior. En M. Theodore and L. Melvin (Eds.) Handbook of Psychology: Personality and Social Psychology, (5). Nueva York: John Wiley y Sons, Inc. XIX.

Blom, F. \& Pereda, N. (2009). Niños y niñas soldado: consecuencias psicológicas e intervención. Barcelona. Anuario de Psicología, 40(3), 329-344.

Bowlby, J. (1976). La separación afectiva. Buenos Aires: Paidós

Bowlby, J. (1986). Vínculos afectivos. Formación, desarrollo y pérdida. Madrid: Morata

Buss, D. M., \& Kenrick, D.T. (1998). Evolutionary Social Psycology. In D. T. Gilbert., S. T. Fiske \& G. Lindzey (Eds), the Handbook of Social Psychology. E the D., Vol. 2, pp. 982-1026). New York: McGraw-Hill.

Buss, D. M., \& Reeve, H. K. (2003). Evolutionay Psychology and Developmental Dynamics: Comment on Lickliter and Honeycutt. Psychological Bulletin, 129, 848-853. 
Caprara, G. V., Alessandri, G., \& Eisenberg, N. (2012). Prosociality: The contribution of Traits, Values, and Self-efficacy Beliefs. xJournal of personality and social psychology, 102(6), 1289-1303.

Caprara, G. V., Steca, P., Zelli, A., \& Capanna, C. (2005). A New Scale for Measuring Adults' Prosocialness. European Journal of psychological assessment, 21(2), 77-89.

Carlo, G., McGinley, M., Hayes, R., Batenhorst, C., \& Wilkinson, J. (2007). Parenting Styles or Practices? Parenting, Sympathy and Prosocial Behaviors among Adolescents. Journal of Genetic Psychology, 168(2), 147-176.

Carlo, G., Mestre, M. V., Samper, P., Tur, A. M., \& Armenta, B. E. (2010). Feelings or Cognitions? Moral Cognitions and Emotions as Longitudinal Predictors of Prosocial and Aggressive Behaviors. Personality and Individual Differences $48,872-877$.

Carrera, P., Caballero, A. \& Oceja, L. V. (2003). Altruismo y conducta prosocial. En D. Páez, I. Fernández, S. Ubillos y E. Zubieta (Cords.), Psicología social, cultura y educación (pp. 605-629). Madrid: Pearson.

Cortes, L., \& Buchanan, M. J. (2007). The experience of Colombian child soldiers from a resilience perspective. International Journal for the Advancement of Counselling, 29, 43-55.

Correa, M.C. (2017). Aproximaciones epistemológicas y conceptuales de la conducta prosocial. Revista Zona Próxima, 27(2), 1-21.

Dovidio, J. F., Piliavin, J. A., Schroeder, D. A. \& Penner, L. A. (2006). The social psychology of prosocial behavior. Mahwah: Erlbaum.

Dugatkin, L. A. (2007). Qué es el altruismo. La búsqueda científica de la generosidad. Madrid: Katz Editores.
Echeburúa, E. (2004). Superar un trauma. El tratamiento de las víctimas de sucesos violentos. Madrid: Pirámide.

Eisenberg, N., \& Fabes, R. A. (1998). Prosocial development. En W. Damon (Series Ed.), N. Eisenberg (Volumen Ed.). Handbook of child psychology: Social, Emotional, and Personality Development (5a ed., vol. 3, pp. 701-778). Nueva York: Wiley

Eisenberg, N., \& Morris, A. S. (2004). Moral Cognitions and Prosocial Responding in Adolescence. En R. Lerner \& L. Steinberg (Eds.), Handbook of Adolescent Psychology (pp. 155188). Nueva York: Wiley.

Eisenberg, N., Cumberland, A., Guthrie, I. K., Murphy, B. C., \& Shepard, S. A. (2005). Age Changes in Prosocial Responding and Mral Reasoning in Adolescence and Early Adulthood. Journal of research in adolescence, 15(3), 235-260.

Eisenberg, N., Fabes, R., Guthries, I., \& Reiser, M. (2000). Dispositional Emotionality and Regulation: their Role in Predicting Quality of Social Functioning. Journal Personality and Social Psychology, 78(1), 136-157.

Eisenberg, N., Valiente, C., \& Champion, C. (2004). Empathy-related Responding. Moral, Social, and Socialization Correlates. En A. G. Miller (Ed.), the Social Psychology of Good and Evil (pp. 386-415). Nueva York: The Guilford Press.

Eisenberg, N., Zhou, Q., \& Koller, S. (2001). Brazilian Adolescents' Prosocial Moral Judgment and Behavior: Relations to Sympathy, Perspective Taking, Gender-role Orientation and Demographic Characteristics. Child development, Estados Unidos 72, 518-534.

Fernández-Pinto, I., López-Pérez B. \& Márquez M. (2008). Empatía: medidas, teorías y aplicaciones en revisión. Anales de Psicología, 24,(2), 284-298. 
Garaigordobil. M. (2005). Diseño y evaluación de un programa de intervención socioemocional para promover la conducta prosocial y prevenir la violencia. Madrid: Ministerio de Educación y Ciencia.

Gómez, A. \& Gaviria, E. (2007). Conducta de ayuda, conducta prosocial y altruismo. Cap 14. En J. F. Morales, M. C, Moya, E. Gaviria, \& I, Cuadrado. (Coord). Psicología Social. (3a . ed.). Madrid: Pearson.

González Portal, M. D. (2000). Conducta prosocial: Evaluación e Intervención. Madrid: Morata

Gómez, A. S., \& Narváez, N. (2017). Conductas prosociales en adolescentes desvinculados de grupos armados ilegales, pertenecientes a la modalidad Hogar Sustituto Tutor del ICBF, en Manizales. En V, Castellanos (comp). Innovaciones en Psicología y Salud Mental. Ponencia presentada en el IV Congreso Internacional y IX Nacional en Tunja.

Gómez, A. S. (2017). Prosocialidad: una propuesta alternativa para el trabajo con niños, niñas y adolescentes desvinculados de grupos armados ilegales. En S.A, Alvarado, \& P.A, Vommaro. (Comp). Infancias y juventudes iberoamericanas: transformaciones democráticas, justicia social y procesos de construcción de paz. Centro Editorial CINDE - Centro de Estudios Avanzados en Niñez y Juventud Fundación Centro Internacional de Educación y Desarrollo Humano, CINDE - Universidad de Manizales - CLACSO

Graziano, W. G., Habashi, M. M., Sheese, B. E., \& Tobin, R. M. (2007). Agreeableness, Empathy, and Helping: A Person Situation Perspective. Journal of Personality and Social Psychology, 93, 583-599.

Greenwald, R. (2005). Child Trauma Handbook. A Guide for Helping Trauma- exposed Children and Adolescents. Binghamton, NY, US The Haworth press.
Gutiérrez San Martín, M., Escartí, A., \& Pascual, C. (2011). Relaciones entre empatía, conducta prosocial, agresividad, autoeficacia y responsabilidad personal y social de los escolares. Psicothema, 23(1), 13-19.

Gutiérrez, F. (2015). ¿Una historia simple? En contribución al entendimiento del conflicto armado en Colombia. Bogotá: Comisión histórica del conflicto y sus víctimas. Ediciones desde abajo.

Hemming, J. (1991). The Psychology of Moral Maturity. Journal of Moral Education 20, 2, 127-137.

Henao, G., Ramírez, C., \& Ramírez, L. (2007). Las prácticas educativas familiares como facilitadoras del proceso de desarrollo en el niño y niña. El Ágora 7(2), 233-240.

Hoffman, M. L. (1992). La aportación de la empatía a la justicia y al juicio moral. En N. Eisenberg, \& J. Strayer (Eds.), La empatía y su desarrollo (pp. 59-93). Bilbao: Desclée de Brouwer.

Holmgren, R., Eisenberg, N., \& Fabes, R. A. (1998). The Relations of Children's Situational Empathy related Emotions to Dispositional Prosocial Behaviour. International Journal of Development, Estados Unidos 22, 169-193.

ICBF, OIM, Unicef. (2014). Impacto del conflicto armado en el estado psicosocial de niños, niñas y adolescentes. Bogotá. Instituto Colombiano de Bienestar Familiar. Organización Internacional para las Migraciones. Fondo de las $\mathrm{Na}$ ciones Unidas para la Infancia

Kärtner, \& Keller. (2010). Cognitive and Social Influences on Early Prosocial Behavior in Two Sociocultural Contexts. Developmental Psychology, 46(4), 905-914.

Kimmel, C. E., \& Roby, J. L. (2007). Institutionalized Child Abuse: The use of Child Soldiers. International Social Work, 50, 740-754. 
Kohlberg, L. (1969). Stage and Sequence: The Ccognitive-developmental Approach to Socialization. En D. A. Goslin (Ed.), Handbook of Socialization Theory and Research. Chicago: Rand McNally.

Kohlberg, L. (1976). Estadios morales y moralización. El enfoque cognitivo-evolutivo. En E. Turiel et al., (1989). El mundo social en la mente infantil. Madrid: Alianza Psicología.

Kohlberg, $L$ (1987). El enfoque cognitivo-evolutivo de la educación moral. En J. A. Jordan y F. F. Santolaria, La educación moral, hoy. Cuestiones y perspectivas. Barcelona: Biblioteca Universitaria de Pedagogía. PPU: pp. 85-115.

Kohlberg, L, Levine, Ch. y Heder, A. (1992). Psicología del Desarrollo Moral. Bilbao: Desclée de Brouwer

Kohlberg, L., Power, F. C., \& Higgins, A. (2002). La educación moral ( $2^{\mathrm{a}}$ ed.). Barcelona: Gedisa.

Latané, B. (1981). The Psychology of Social Impact. American psychologist, 36, 343-356

Latané, B., \& Darley, J. M. (1970). The Unresponsive Bystander. Why Doesn't'the help? New York: Appleton-Century-Crofts.

Latané, B., \& Darley, J. M. (1976). Help in a crisis: bystander response to an emergency. In J. W. Thibaut \& J.T Spence (Eds). Contemporary Topics in Social Psychology (pp. 309-332). Morristown, Nj: General Learning Press

López, F., Apodaca, P., Etxebarría, I., Fuentes, M. J. \& Ortiz, M. J. (1998). Conducta prosocial en preescolares. Infancia y Aprendizaje, 82, 45-61.

Machel, G. (1996). Impact of Armed Conflict on Children. New York, NY: United Nations.

Maclurea, R. \& Denov, M. (2006). I didn't Want to Die so I Joined them: Structuration and the Process of Becoming boy Soldiers in Sie- rra Leone, Terrorism and Political Violence, 18,(1), 119-135.

Mago, I. (2009). ¿De niño combatiente a ciudadano? Los retos de la reintegración política de niños desvinculados del conflicto armado colombiano. Bogotá. Universidad de Los Andes.

Martí Vilar, M. (2010). Razonamiento moral y prosocialidad. Fundamentos. Madrid: Ccs.

Martí Vilar, M. (Octubre, 2011). Bases teóricas de la prosocialidad. En E. Figueroa (Presidente), Educación para la responsabilidad social: Estrategias de enseñanza y evaluación. Conferencia llevada a cabo en el IV Congreso Internacional en la Universidad de Concepción, Concepción, Chile.

Martí Vilar, M. y Lorente, S. (2010). Factores Determinantes de las conductas prosociales. En M. Martí Vilar. Razonamiento Moral Y Prosocialidad. Fundamentos. Madrid: Ccs.

Martínez, J.G; Barreto, K; Duran, W \& Castro, Y. (2014). Relación entre desarrollo moral y el rol del agresor en situaciones de Bullying. Universidad Sergio Arboleda

Mehlinger, H. (1985). School Practices in moral education.traducido en: Cañón, C. (1985). El sentido de los valores. Teoría, investigación, y sus aplicaciones a la psicología y a la educación. Bogotá: Universidad Javeriana

Mestre, M. V., Frías, M. D., \& Samper, P. (2004). La medida de la empatía: análisis del Interpersonal Reactivity Index. Psicothema, 16, 255-260.

Mestre, V., Frías, D., Samper, P., \& Nácher, M. J. (2003). Estilos de crianza y variables personales como factores de riesgo de la conducta agresiva. Revista Mexicana de Psicología, 20(2), 189-199.

Mestre, V., Samper, P., \& Frías, D. (2004). Personalidad y contexto familiar como factores 
predictores de la disposición prosocial y antisocial de los adolescentes. Revista Latinoamericana de Psicología, 36(3), 445-447.

Mestre, V., Samper, P., \& Frías, M. D. (2002). Procesos cognitivos y emocionales predictores de la conducta prosocial y agresiva: La empatía como factor modulador. Psicothema, 14(2), 227-232.

Mestre, M. V., Samper, P., Frías, M. D., \& Tur, A. M. (2009). Are Women more Empathetic than Men? A Longitudinal Study in Adolescence. The Spanish Journal of Psychology 12(1), 76-83.

Mestre, V., Samper, P., Tur, A. M., Cortés, T. y Nácher, M. J. (2006). Conducta prosocial y procesos psicológicos implicados: un estudio longitudinal en la adolescencia. Revista Mexicana de Psicología, 23(2), 203-215.

Mestre, M. V., Tur, A., Samper, P., Nácher, M.J., \& Cortés, M.T. (2007). Estilos de crianza en la adolescencia y su relación con el comportamiento prosocial. Revista Latinoamericana de Psicología, 39(2), 211-225.

Ministerio de la Protección Social. (2007). Promoción de comportamientos prosociales para la identificación y prevención de la agresión en la primera infancia: Manual para Agentes Educativos Socializadores - AES.

Moñivas, A. (1996). La conducta prosocial. Cuadernos de Trabajo Social, 9, 125-142.

Moreno, M. (2009). Consideraciones sobre el paso a la vida civil de jóvenes desvinculados de grupos armados ilegales. Revista Científica Guillermo de Ockham, 7(2), 65- 74.

Parra, E. I. (2012). Habilidades mentalistas y conducta prosocial en niños escolarizados. Salud Uninorte, 28(1), 113-130.

Pastorelli, C. (2015). Prosocialidad y paz: teoría, investigación e intervención. En G. Tama- yo (presidencia), Programa de Psicología de la Facultad de Ciencias Sociales y Humanas. Conferencia llevada a cabo en la Universidad de Manizales, Manizales, Colombia.

Pécaut, D. (2001). Guerra contra la sociedad. Bogotá: Espasa

Piliavin, J. A., \& Charng, H.W. (1990). Altruism: A Review of Recent Theory and Research. Annual Review of Sociology, 16, 27-65.

Ramírez, M. (2007). Los padres y los hijos: variables de riesgo. Educación y Educadores 10(1), 27-37.

Rest, J. R, Narváez, D., Bebeau, M. J., \& Thoma, S. J. (1999). A Neo-Kohlbergian approach: The Dit and Schema Theory. Educational Psychology Review, 11(4), 291-324.

Rest, J. R. (1986). Moral development: Advances in Research and Theory. New York: Publishers Praeger.

Rethmann, A. (2010). Condenados al silencio, jóvenes excombatientes en Colombia. En Independencias - dependencias - interdependencias, VI Congreso CENSAL, Tolouse, Francia.

Richaud de Minzi, M. C. (2009). Influencia del modelado de los padres sobre el desarrollo del razonamiento prosocial en los/las niños/as. Interamerican Journal of Psychology, 43(1), 187-198.

Richaud de Minzi, M. C., Lemos, V., \& Mesurado, B. (2011). Relaciones entre la percepción que tienen los niños de los estilos de relación y de la empatía de los padres y la conducta prosocial en la niñez media y tardía. Avances en Psicología Latinoamericana, 29(2), 330-343.

Richaud, M. C. (2014). Algunos aportes sobre la importancia de la empatía y la prosocialidad en el desarrollo humano. Revista Mexicana de Investigación en Psicología, 6(2), 171-176. 
Roche. (2010). Prosocialidad: Nuevos desafíos: métodos y pautas para la optimización creativa del entorno. Buenos Aires: Ciudad Nueva.

Romero, Y., \& Chavez, Y. (Junio 2008). El juego de la guerra, niños, niñas y adolescentes en el conflicto armado Colombia. Bogotá. Tabula Rasa, 8.

Springer, N. (2010). ¿Negociar la paz o hacer justicia? Bogotá: Aguilar

Springer, N. (2012). Como corderos entre lobos. Del uso y reclutamiento de niñas, niños y ado- lescentes en el marco del conflicto armado y la criminalidad en Colombia. Bogotá: Naciones Unidas.

Stevens, J. R., Cushman, F.A., \& Hauser, M. D (2005). Evilving the Psychological Mechanisms for Cooperation. Anual Review of Ecology, Evolution and Systematics, 36, 499-518.

Turiel, E. (1979). El desarrollo moral. Madrid: Morata.

Vaughan, G. M., \& Hogg, M. A. (2010). Psicología Social. (5a. ed). Madrid: Panamericana 